
T. DREIER ${ }^{1, \infty}$
B. BOUGIE ${ }^{2}$
N. DAM $^{2}$
T. GERBER ${ }^{1}$

\title{
Modeling of time-resolved laser-induced incandescence transients for particle sizing in high-pressure spray combustion environments: a comparative study
}

\author{
${ }^{1}$ Reaction Analysis Group, Department of General Energy, Paul Scherrer Institute, \\ 5232 Villigen PSI, Switzerland \\ 2 Radboud University Nijmegen, Institute for Molecules and Materials, Applied Molecular Physics, \\ Toernooiveld 1, 6525 ED Nijmegen, The Netherlands
}

\begin{abstract}
Received: 22 December 2005/Revised version: 3 March 2006 Published online: 19 April 2006 • (C) Springer-Verlag 2006
\end{abstract}

ABSTRACT In this study experimental single-pulse, timeresolved laser-induced incandescence (TIRE-LII) signal intensity profiles acquired during transient Diesel combustion events at high pressure were processed. Experiments were performed between 0.6 and $7 \mathrm{MPa}$ using a high-temperature high-pressure constant volume cell and a heavy-duty Diesel engine, respectively. Three currently available LII sub-model functions were investigated in their performance for extracting ensemble mean soot particle diameters using a least-squares fitting routine, and a "quick-fit" interpolation approach, respectively. In the calculations a particle size distribution as well as the temporal and spatial intensity profile of the heating laser was taken into account. For the poorly characterized sample environments of this work, some deficiencies in these state-of-the-art data evaluation procedures were revealed. Depending on the implemented model function, significant differences in the extracted particle size parameters are apparent. We also observe that the obtained "best-fit" size parameters in the fitting procedure are biased by the choice of their respective "first-guess" initial values. This behavior may be caused by the smooth temporal profile of the LII cooling curve, giving rise to shallow local minima on the multi-parameter least squares residuals, surface sampled during the regression analysis procedure. Knowledge of the gas phase temperature of the probed medium is considered important for obtaining unbiased size parameter information from TIRE-LII measurements.

PACS 42.62.-b; 51.30.+i; 82.20.Wt

\section{$1 \quad$ Introduction}

Due to increased worldwide environmental air pollution regulation standards there is growing interest in quantitative information on the amount and size ranges of exhaust particulates emitted during engine combustion. A reduction of exhaust emissions in Diesel engines requires a better basic understanding of in-cylinder soot formation and oxidation mechanisms to realize optimized combustion strategies within a wide range of engine operating conditions. Nonintrusive, in-situ optical measurement of, e.g., soot particle

Fax: +41-56-310-2199, E-mail: thomas.dreier@psi.ch size and volume fraction provides such information, and may further enable a direct validation of kinetic mechanisms needed to understand in-cylinder soot formation and oxidation processes. One powerful soot diagnostic technique with high temporal and spatial resolution is laser-induced incandescence (LII) [1], where particulate matter is heated to high temperatures via absorption of a pulsed laser beam, and its subsequent incandescence during cooling is recorded by a fast photodetector. For a recent review of the LII technique and its applications in combustion diagnostics see [2] and references cited therein. For scalar field measurements the soot cloud is intersected by a thin laser sheet and incandescence radiation is imaged on a CCD-camera allowing two-dimensional spatially resolved soot volume fraction diagnostics with high temporal resolution. In time-resolved LII (TIRE-LII) [3, 4] the soot blackbody radiation is recorded during the particle heatup and cooling phase. Particle size information can then be obtained from a best-fit comparison of experimental temporal signal decay curves and those calculated from the particles energy and mass balance equations.

Despite many demonstrations of TIRE-LII for particle sizing, data evaluation still remains controversial. In the present work a comparative study was undertaken to deduce particle size information from TIRE-LII measurements at high pressure to address some issues concerning the applied fitting procedures and the model functions involved in the theoretical description of the process. For this purpose three submodels proposed in the current literature were used for treating experimental LII signal profiles via two data evaluation strategies: a nonlinear least-squares fit of the full LII decay curve, and a "quick-fit" method, where the measured signal trace is approximated piecewise by simple exponential decay functions.

Laser-induced incandescence has previously been used for soot diagnostics in high pressure environments, and in some cases mean particle diameters have been determined from TIRE-LII measurements in laminar flames [5] as well as in small and heavy duty optically accessible Diesel engines [6-9]. In the present work the mentioned data evaluation procedures were applied for LII signal profiles obtained during transient Diesel combustion events in a high pressure constant volume cell, and in a heavy-duty truck engine, covering a large pressure range. The purpose is to highlight some limitations of LII particle sizing in situations where 
a full characterization of the thermophysical properties of the probed environment is hardly possible.

\section{Theoretical approach \\ $2.1 \quad$ LII submodels}

Several publications present physical models to describe the temporal evolution of the LII signal during and after laser-induced particle heating. All three approaches employed in this study originate from the pioneering work of Melton [1], who first investigated the potentials of LII for in-situ soot diagnostics. In this treatment the mass- and energy-balance equations for a laser-heated particle, i.e. absorption of radiation and subsequent heat loss due to vaporization, conduction and radiation can be written as,

$$
\begin{aligned}
& \frac{\mathrm{d} m_{\mathrm{p}}}{\mathrm{d} t}=4 \pi r_{\mathrm{p}}^{2} \varrho_{\mathrm{v}} u_{\mathrm{v}} \\
& \frac{\mathrm{d} U}{\mathrm{~d} t}=\dot{Q}_{\mathrm{abs}}-\dot{Q}_{\mathrm{vap}}-\dot{Q}_{\mathrm{con}}-\dot{Q}_{\mathrm{rad}},
\end{aligned}
$$

where the rate of change of the particles internal energy $U$ is given by

$\frac{\mathrm{d} U}{\mathrm{~d} t}=\frac{\mathrm{d}\left(m_{\mathrm{p}} c_{\mathrm{s}} T\right)}{\mathrm{d} t}$.

In (1) $\varrho_{\mathrm{v}}$ and $u_{\mathrm{v}}$ designate the density and thermal speed of vapour molecules leaving the surface of a soot particle with specific heat $c_{\mathrm{S}}$ and temperature $T$, respectively. The expressions in (2) account for heat flux (in Watt) due to absorbed energy, and loss processes due to vaporization, heat conduction and radiation, respectively. The particle mass $m_{\mathrm{p}}$ is given by $m_{\mathrm{p}}=\frac{4}{3} \pi r(t)^{3} \varrho_{\mathrm{s}}$, with $\varrho_{\mathrm{s}}$ the density of soot.

It was shown by Melton that the temporal decay of the LII signal trace is a direct measure of particle size [1]. This is accomplished by solving (1)-(3) for particle temperature and radius as time varying functions for a calculation of the LII signal response. In the present work three later versions of this approach are compared when coupled to optimization procedures for obtaining particle size information, i.e., within a least-squares minimization routine. For a closer description of these models the reader is referred to the original literature $[4,7,10,11]$. One should mention here that more sophisticated models exist, which take into account additional physical-chemical processes, such as nonthermal photodesorption, oxidative heating, particle melting, annealing and others [12] relevant for low and high laser fluence.

The main differences in the cited derivations for the LII process $[4,7,10,11]$ concern model-specific parameters and the temperature dependence of individual thermodynamic functions employed in the individual heat loss terms, such as soot vapor pressure, evaporation enthalpy, specific heat, etc. Table 1 collects several parameters inherent in each of the employed model functions as cited in the original publications. Most physical parameters are similar in magnitude; however some differ in the assumption of a temperature and pressure dependence within ranges relevant for the present application. For illustration, Fig. 1 depicts the soot vapour pressure, $p_{\text {vap }}$, and the sublimation enthalpy, $\Delta H_{\text {vap }}$, employed in each sub-model for temperatures typical in LII experiments. The same dependencies are also plotted for the recently published model of Smallwood et al. [13] (dot-dashed lines). It is obvious that - in between models - considerable variations in these functional dependencies exist, which, in case of significant sublimation, will entail a different temporal behaviour of the calculated LII transient profiles. If particle temperature can not be specified another critical parameter in the calculations is the complex soot refractive index, which affects the particles absorption and emission characteristics [14].

Thermophoretic probe sampling in laminar and turbulent sooting flames, coupled with high resolution electron microscopy [15-17] suggests a close to lognormal size distribution (PSD) function for the primary particle radius $r$,

$P(r)=\frac{1}{\sqrt{2 \pi} \sigma r} \exp \left[-\frac{\left(\ln r-\ln r_{\mathrm{m}}\right)^{2}}{2 \sigma^{2}}\right]$,

an assumption also made in the present calculations. In (4) $r_{\mathrm{m}}$ denotes a count median particle radius and $\sigma=\ln \sigma_{\mathrm{g}}$ the $\log$ -

\begin{tabular}{|c|c|c|c|}
\hline Parameter & $\begin{array}{l}\text { Model } 1 \\
\text { (Bockhorn et al. [10]) }\end{array}$ & $\begin{array}{l}\text { Model } 2 \\
\text { (Schittkowski et al. [11]) }\end{array}$ & $\begin{array}{l}\text { Model } 3 \\
\text { (Roth et al. }[4,7])\end{array}$ \\
\hline Internal U & Constant $\rho, \mathrm{Cs}$ & Constant $\rho, \mathrm{Cs}$ & Constant $\rho, T$-dependent $\mathrm{Cs}, \mathrm{Cp}$ \\
\hline Absorption & $m=1.90-0.55 i$, Rayleigh & $m=1.90-0.55 i$, Rayleigh & $m=1.57-0.56 i$, Rayleigh \\
\hline Radiation & Constant $m$, Rayleigh & Constant $m$, Rayleigh & Constant $m$, Rayleigh \\
\hline \multirow[t]{5}{*}{ Sublimation } & Kinetically controlled & Kinetically controlled & Transition/continuum regime \\
\hline & $\alpha_{\mathrm{m}}=1$ & $\alpha_{\mathrm{m}}=1$ & $\alpha_{\mathrm{m}}=1$ \\
\hline & Only $C_{3}$ sublimes & Only $C_{2}$ sublimes & Only $C_{3}$ sublimes \\
\hline & $P_{\text {vap }}(T)$ : Antoine equation & $P_{\text {vap }}(T)$ : Clausius equation & $P_{\text {vap }}(T)$ : Clausius equation \\
\hline & $H_{\mathrm{vap}}(T)=$ constant & $\begin{array}{l}H_{\mathrm{vap}}(T)=6.579 \times 10^{7} \mathrm{~T}- \\
0.5364 \mathrm{~J} / \mathrm{mol}\end{array}$ & $H_{\mathrm{vap}}(T)=$ constant \\
\hline \multirow[t]{2}{*}{ Conduction } & $\begin{array}{l}\text { Free molecular regime } \\
\alpha_{\mathrm{T}}=0.2\end{array}$ & $\begin{array}{l}\text { Transition regime } \\
\alpha_{\mathrm{T}}=0.9\end{array}$ & $\begin{array}{l}\text { Free molecular/transition/ } \\
\text { continuum regime }\end{array}$ \\
\hline & & $\begin{array}{l}T \text {-dependent: heat transfer } \\
\text { coefficient } \\
\text { thermal conductivity } \\
\text { mean free path }\end{array}$ & $\alpha_{\mathrm{T}}=0.23$ \\
\hline
\end{tabular}
arithm of its geometric standard deviation $\sigma_{\mathrm{g}}$. We are aware

TABLE 1 Set of input parameters as implemented in the sub-model approaches 1 [10], 2 [11] and 3 [4, 7], respectively, and used in this work for calculating the LII signal response. $U$ : internal energy, $m, \rho$ : soot refractive index, density, $C s, C p$ : heat capacity of soot and air, respectively, $p_{\mathrm{vap}}, \Delta H_{\mathrm{vap}}$ : soot vapor pressure, sublimation enthalpy, $\alpha_{\mathrm{m}}, \alpha_{\mathrm{T}}$ : mass and energy accommodation coefficient, respectively 




FIGURE 1 Temperature dependence of soot vapor pressure ( $\left.p_{\text {vap }}\right)$ and sublimation enthalpy $\left(\Delta H_{\mathrm{vap}}\right)$ as implemented in the various LII model functions employed in this work. Solid line: model 1 [10]; dashed line: model 2 [11]; dotted line: model $3[4,7]$. For comparison, the same dependencies in the model of Smallwood et al. [14] are also shown as a dot-dashed line

of this oversimplification of the morphology and size distribution of real engine or flame generated soot as revealed in numerous experimental $[18,19]$ and modelling $[20]$ work in the literature, and this has consequences with respect to heat transfer between particles and the surrounding gas [21]. However, since these assumptions are made in all three models here for calculating the LII response and as only relative comparisons are made, this simplification is justified.

\section{$2.2 \quad$ LII response and particle size information}

In all models considered here absorption and scattering is treated in the Rayleigh limit [1]. This assumption may no longer hold for larger spherical particles and especially aggregates typically formed in flame soot [22]. However, since no changes to the models are done in this investigation we stick to this simplification for the sake of simplicity and consistency. For comparison with experiments the calculated LII signal trace was convoluted with a measured instrument response function to account for the finite temporal resolution of the detection system (consisting of a photomultiplier and a digital storage oscilloscope). In practical measurements particles are exposed to different irradiation intensities corresponding to the spatial variation of laser fluence across the beam in the focal volume. To account for this non-uniform particle heating, a procedure similar to [23] was applied by solving (1)-(3) for a number of fluence values along the diameter of an assumed Gaussian beam profile, and weighting each contribution to the total LII signal intensity with the respective fractional beam cross section. The quality of the Gaussian beam shape was, however, not closely characterized experimentally. The total LII time response was then calculated by summing the signal intensities from each beam segment using a trapezoidal integration method.

Particle size information was extracted from measured LII transient signals via two approaches. The first made use of the commonly implemented full least-squares curve fit of calcu-

lated LII model functions to experimental decay profiles using a Levenberg-Marquart algorithm [24]. Variables to be optimized in the fit were the PSD-function parameters $r_{\mathrm{m}}$ and $\sigma$ from (4), as well as the initial soot temperature right after peak laser intensity for a global minimization of the sum of leastsquares residuals,

$\chi^{2}=\sum_{i=1}^{N}\left(S_{\exp }(i)-S_{\text {theo }}(i, a)\right)^{2}$.

In this equation $S_{\text {exp }}(i)$ and $S_{\text {theo }}(i, a)$ are the measured and calculated LII signal intensity values at time $t_{i}$ and $a$ is a parameter vector of several fit components. Other parameters in the calculation were kept fixed during the fitting procedure, because they either were known from experiments (gas pressure, $p_{\mathrm{g}}$ ), or must be estimated (gas temperature, $T_{\mathrm{g}}$ ). In the second approach a "quick-fit" method proposed by Dankers et al. [25] was applied, in which single-exponential decay functions were fitted to experimental LII decay profiles within two time intervals, one located close to the initial peak, the other in the slower decay portion in the tail of the signal trace. This strategy should account for the different contributions from small and large particles within the size distribution to the cooling behavior, i.e., during the early and late portions, respectively, of the LII signal trace. The two parameters $r_{\mathrm{m}}$ and $\sigma$ in the PSD-function $P(r)$ were then determined by a table look-up in a pre-calculated library of decay rate pairs evaluated from single exponential fits to model functions for a suitable range of experimental variables, including PSD-function parameters, gas pressure and temperature.

\section{$3 \quad$ Experimental procedures}

LII temporal profiles were obtained in our own experiments on two separate high pressure turbulent combustion environments: the constant volume spray combustion chamber at the Paul Scherrer Institute (PSI), and the heavyduty truck Diesel engine located at Radboud University of

Constant Volume Cell (PSI)

Nozzle

Injection pressure Injection duration

Pressure at time of measurement $T_{\text {gas }}$

Excitation wavelength Detection wavelength

\begin{tabular}{lc}
\hline \multicolumn{2}{c}{ Heavy-duty engine (RU) } \\
\hline Displacement & $11.6 \mathrm{~L}$ \\
Head & $4-$ Valve \\
Speed & $1430 \mathrm{rpm} @ 25 \%$ load \\
Nozzle & 8 -hole: $\varnothing 0.224 \mathrm{~mm}$ \\
Injection pressure & $45 \mathrm{MPa}$ max. (line pump) \\
Pressure at time of measurement & $1.6-7 \mathrm{MPa}$ \\
Excitation wavelength & $1064 \mathrm{~nm}$ \\
Detection wavelength & $(550 \pm 10) \mathrm{nm}$
\end{tabular}

TABLE 2 Main operating conditions of the constant volume high temperature, high pressure spray combustion cell, and the heavy-duty truck Diesel engine operated at PSI and RUN, respectively. Details for the respective LII experiments are also given 
Nijmegen (RU). The main features of each test facility are listed in Table 2. A brief outline and mode of operation of each setup is given below, more detailed descriptions can be found in previous publications [26, 27].

The heatable constant volume spray combustion chamber at PSI can be operated at temperatures and pressures up to $800 \mathrm{~K}$ and $8 \mathrm{MPa}$, respectively. Preheated and pressurized air is loaded from an autoclave through a valve-operated inlet port. Optical access to the cubic combustion chamber perpendicular to the fuel spray propagation direction is provided by four sapphire windows with $40 \mathrm{~mm}$ clear aperture in each side wall. A computer controlled measurement cycle is initiated by opening the gas supply valve and filling the combustion chamber at the desired temperature and pressure in less than $35 \mathrm{~ms}$ by activating the inlet valve with a flywheel-driven camshaft. After a fixed time delay after the inlet valve closure Diesel fuel is introduced through a solenoid-activated single-hole fuel injector at pressures between 50 and $70 \mathrm{MPa}$. After the ignition and combustion events have ceased the hot gases are expelled through the pneumatically activated exhaust valve. A camshaft encoder and pulse coincidence detector electronics synchronize the cell cycle with external measurement devices (LII excitation laser, oscilloscope). The gas pressure inside the combustion chamber is recorded with a water cooled pressure sensor (Kistler).

At Radboud university experiments were conducted on an optically accessible cylinder of a six-cylinder, heavy-duty Diesel engine running with a commercial low-sulphur Diesel fuel at about $25 \%$ load. The laser and detection system were triggered by a crankshaft encoder. Optical access for the laser beam is possible via quartz windows in the cylinder top and the piston crown. A slot in the piston in combination with a window in the side of the cylinder provides access to the combustion process from the side.

In both experiments soot incandescence was generated using the unseeded fundamental output $(1064 \mathrm{~nm})$ of a Nd:YAG-laser, with pulse energies around $15 \mathrm{~mJ}$ (at PSI: $10^{3} \mathrm{~J} / \mathrm{m}^{2}$ in a $15 \mathrm{~mm}$ wide and $0.5 \mathrm{~mm}$ thick laser sheet) and $9 \mathrm{~mJ}$ (at RU: $500 \mathrm{~J} / \mathrm{m}^{2}$, circular beam cross section), respectively. Spectral filtering of the detected LII radiation was necessary to suppress interfering flame induced soot incandescence and chemiluminescence emissions (see Table 2).

In both test facilities laser diagnostics suffer from a highly turbulent, non-stationary combustion environment with large density gradients and light attenuation affecting both the incoming laser beam and the detected signal radiation. For a meaningful interpretation of the LII time profiles all measurements reported here were done in single-pulse mode for selected time delays after the start of injection (SOI) or crank angle position, respectively.

\section{$4 \quad$ Results}

\subsection{Comparison of model functions}

In the following paragraphs some general aspects of the calculated LII response are illuminated. Using model 1 [10] Fig. 2 presents the temporal evolution after laser heating (at time zero) of the main individual contributions in the heat flux equation (2), $Q_{\text {con }}, Q_{\text {vap }}, Q_{\text {rad }}$, as well as the LII signal profiles calculated for three typical gas pressures, a gas temperature of $1900 \mathrm{~K}$ and a mean particle diameter of $20 \mathrm{~nm}$. One observes that with increasing pressure energy loss by heat conduction gains in importance, but also drops off faster with time during cooling of the particle ensemble. Due to the low laser fluence case considered here, the vaporization term at peak temperature is already smaller by two orders of magnitude, and it drops faster at higher pressures as a consequence of the non-linear dependence of soot vapour pressure with temperature.

Integrating similar temporal profiles as depicted in Fig. 2 between 5 and $200 \mathrm{~ns}$ after their respective peak values at several pressures using sub-models 1 and 3 , respectively, leads to the dependencies illustrated in Fig. 3. The calculations were performed assuming a gas temperature of $1800 \mathrm{~K}$, and size distribution parameters in (4) of $r_{\mathrm{m}}=30 \mathrm{~nm}$ and $\sigma_{\mathrm{g}}=1.4$. In this case, the thermal energy accommodation coefficient $\alpha_{\mathrm{T}}$ in Table 1 was taken as one for both model functions. The comparison shows a slower LII signal intensity drop with increasing pressure for sub-model 3 , which is considered to be mainly due to its smaller heat conduction term in the pressure regime investigated here (lower panel in Fig. 3).

\subsection{Particle size information from full LSQ curve fits}

Each sub-model was tested using the least-squares fitting strategy with variation of some initial guess values for the particle size parameters (mean particle radius, $r_{\mathrm{m}}$, geometric standard deviation $\sigma$ of the assumed lognormal PSDfunction) and gas temperature. The regression analysis was applied to the same LII data sets from the cell and engine

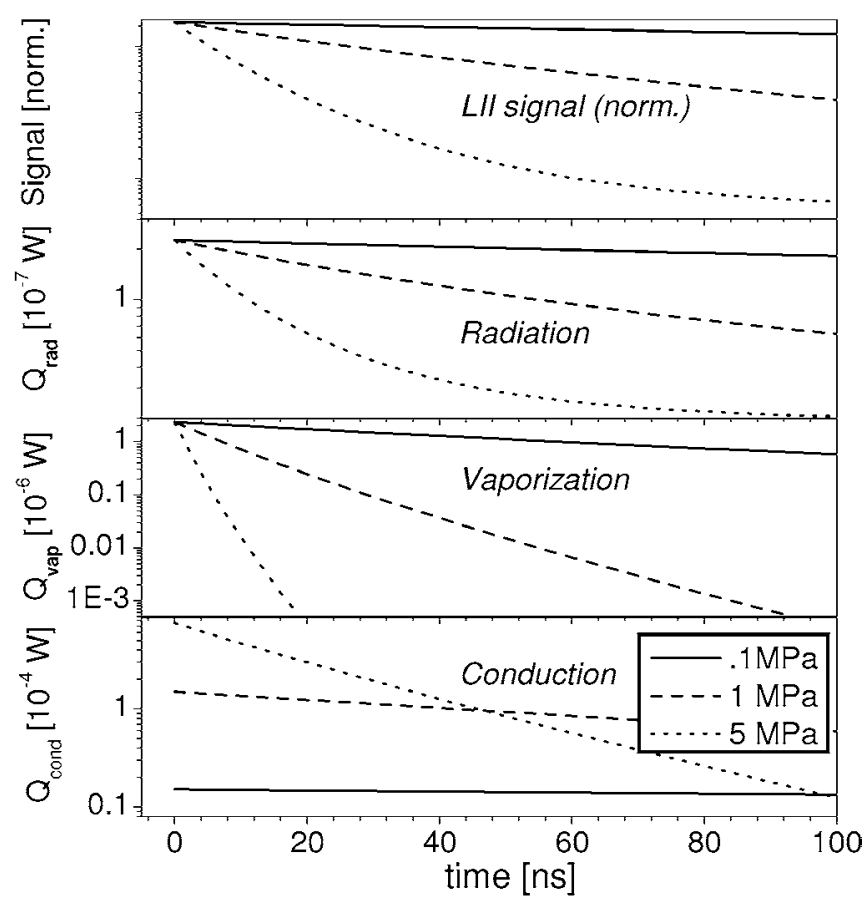

FIGURE 2 Calculated time dependence of heat flux $W$ due to conduction ( $\left.Q_{\text {cond }}\right)$, vaporization $\left(Q_{\text {vap }}\right)$ and radiation $\left(Q_{\text {rad }}\right)$, respectively, as well as the calculated LII signal trace for a laser-heated particle ensemble characterized by a lognormal size distribution with a mean particle diameter $d_{\mathrm{m}}=20 \mathrm{~nm}$ and a distribution width of $\log \sigma_{\mathrm{g}}=0.34$, using model function 1 [10]. Parameter: gas pressure. Other fixed parameters: $T_{\text {gas }}=1900 \mathrm{~K}, T_{\text {soot }}=3400 \mathrm{~K}$ 




FIGURE 3 Evolution with gas pressure of time-integrated (5-200 ns after peak values) heat flux [Ws] of a laser-heated particle ensemble due to conduction $\left(Q_{\text {cond }}\right)$ and evaporation ( $\left.Q_{\text {vap }}\right)$, and LII signal intensity, respectively, using sub-model 1 [10] (solid lines) and 3 [4, 7] (dashed lines). Other fixed parameters as in Fig. 2, with $r_{\mathrm{m}}=30 \mathrm{~nm}$

measurements, respectively, using the recorded gas pressures at the time of measurement. A previous analysis of LSQ residuals evaluated between the fit-functions and a best-fit LII signal curve for a range of laser-induced initial soot temperatures right after the laser pulse, revealed that this parameter does not have a large influence on the deduced particle size [6]. Therefore, this quantity was estimated from the average laser fluence [4] in the experiments and kept fixed at $3400 \mathrm{~K}$ in all test runs. Using sub-model 3 in the modeling calculation procedures two LII sample files from the cell and engine experiments, respectively, are depicted in Fig. 4 (circles) together with the fitted decay curves (solid lines). The equal time spans in both plots highlight the shortening of the temporal decay profile with increasing pressure. The normalized individual instrument response functions in both experiments as measured with residual $532 \mathrm{~nm}$ stray light from the $\mathrm{Nd}$ :YAG laser are also shown (dashed lines). Due to fast particle cooling in the high pressure environments the convolution of the calculated LII transients with the measured temporal instrument response functions was essential to adequately model the initial part of the decay curves.

Using sub-model 3 Fig. 5 displays the best-fit particle size parameters - mean particle radius $r_{\mathrm{m}}$ (upper panel) and distribution width $\ln \sigma_{\mathrm{g}}$ (lower panel) - extracted in the LSQ fitting routine for the data acquired in the cell and engine experiments, respectively. To illustrate some possible trends with pressure and "first-guess" initial values, results are presented for three combinations of the initial-guess parameters particle radius $\left(r_{\mathrm{m}}^{0}\right)$, distribution width $\left(\ln \sigma_{\mathrm{g}}^{0}\right)$, and gas temperature $\left(T_{\mathrm{g}}^{0}\right)$. In each plot the first seven bar triplets represent data

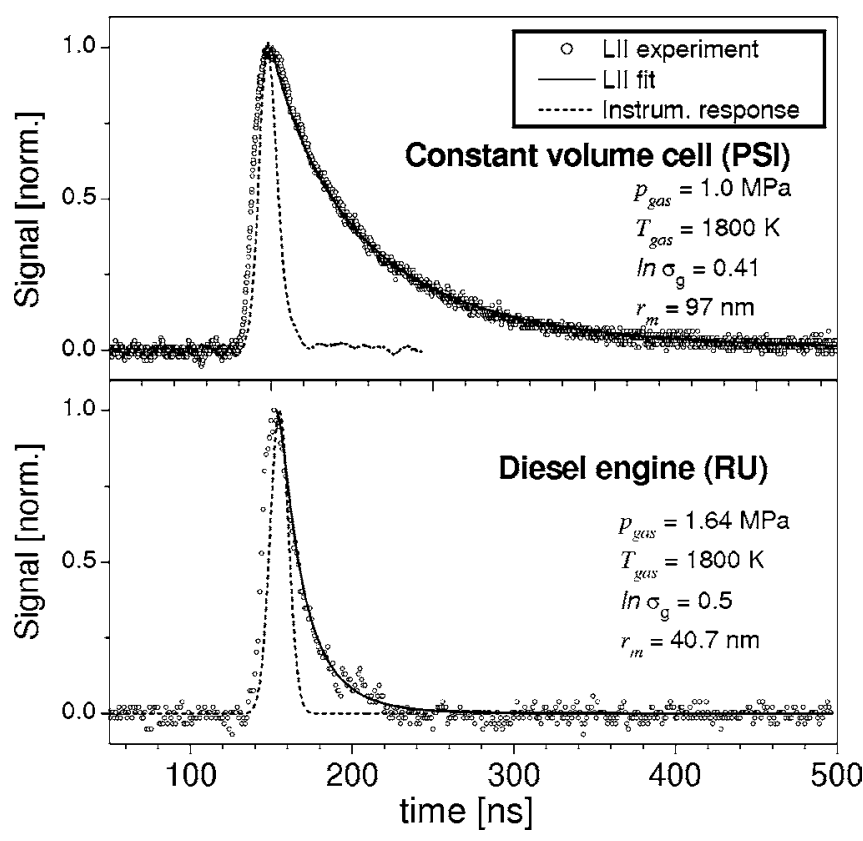

FIGURE 4 Experimental LII signal traces (circles) obtained at the indicated gas pressures in the constant volume spray combustion cell (upper panel) and in the Diesel engine (lower panel), together with a best-fit decay curve (solid lines) using sub-model $3[4,7]$. The temporal response functions of the respective excitation/detection systems are also shown as dashed lines in each plot. The best-fit mean particle radius $\left(r_{\mathrm{m}}\right)$, size distribution width $\left(\sigma=\ln \sigma_{\mathrm{gp}}\right)$ and the initial gas temperature in the fit are also displayed

from the cell, while the last three (in the grey-shaded segment of both panels) are from the engine measurements. It is observed that for the same initial gas temperature (black and grey bars), if the initial-guess particle radius is lowered from 50 to $30 \mathrm{~nm}$ the obtained best-fit radius also decreased, while at the same time the best-fit $\sigma$-value increases. Similarly, when the "first-guess" gas temperature is increased for the same $\left(r_{\mathrm{m}}^{0}, \ln \sigma_{\mathrm{g}}^{0}\right)$-pair the best-fit particle radius further declines (set of grey vs. white bars), but the best-fit $\sigma$-value shows no obvious trend. In this case a higher gas temperature means a reduction of the temperature gradient between particle and environment, i.e., the particle cooling rate in the sample, which is compensated in the fit by a reduction in mean particle size. An even stronger bias in the dependence of bestfit parameter values with their first-guess input is found for models 1 and 2 .

It is beyond the scope of this study to discuss absolute values of the extracted particle size parameters in view of their development with pressure and of possible soot formation mechanisms in the respective combustion environments. Rather, the purpose here is to recognize general trends in the outcome of the commonly implemented data analysis procedures in LII particle sizing and the use of various sub-model functions.

Figure 6 collects, for a particular set of "initial-guess" parameters $T_{\mathrm{g}}^{0}, r_{\mathrm{m}}^{0}$, and $\sigma_{\mathrm{g}}^{0}$, the best-fit particle radius, $r_{\mathrm{m}}$, evaluated from the LSQ analysis of the cell and engine measurements, respectively, using model functions 1-3. It is observed that for all models $r_{\mathrm{m}}$ follows a similar trend with gas pressure, except that its absolute magnitude varies considerably for the different model functions used. This finding emphasizes that the choice of suitable thermophysical parameters adapted to 


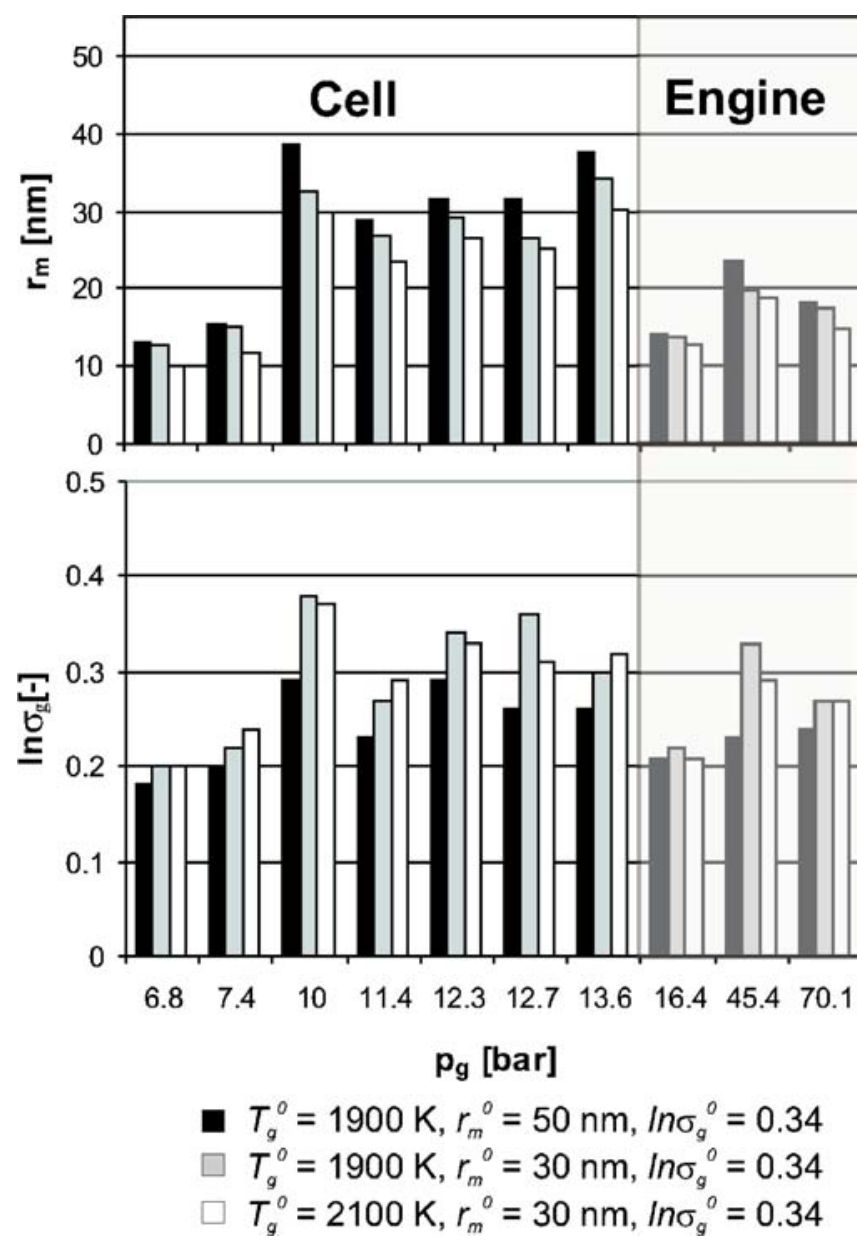

FIGURE 5 Best-fit mean particle radii $\left(r_{\mathrm{m}}\right)$ and distribution widths $\left(\ln \sigma_{\mathrm{g}}\right)$ extracted from the least squares fitting of experimental LII signal traces obtained at the indicated pressures in the constant volume cell (left 7 bar triplets) and in the Diesel engine (right 3 bar triplets) using sub-model $3[4,7]$. For each pressure three best-fit results are displayed as separate grayscale bars using a different combination of initial start parameters for gas temperature $T_{\mathrm{g}}^{0}$, particle radius $r_{\mathrm{m}}^{0}$, with distribution width $\ln \sigma_{\mathrm{g}}^{0}=0.34$

the experimental situation is critical for unambiguous LII particle sizing.

\subsection{Particle size information from quick-fit methods}

A conceptually simple approach for particle sizing by time-resolved LII has been proposed by Dankers et al. [25], where the measured signal curve is fitted with single exponential decay functions, usually in two time windows with visibly different temporal behaviour. This usually is the case at early and late times after the peak signal. At times right after the peak of the signal (hereafter termed early decay, with rate $k_{1}$ ) small particles, if their number density is large enough, may contribute significantly to the LII intensity, whereas at later times (here termed late decay, with rate $k_{2}$ ) cooling of larger particles dominates the signal leading to a slower decay. Particle size information can then be obtained by comparison of such decay rate pairs with a library of pre-calculated decay rates determined from corresponding LSQ fits of exponential functions to artificial LII signal traces for a range of experimental conditions (gas pressure, tem-

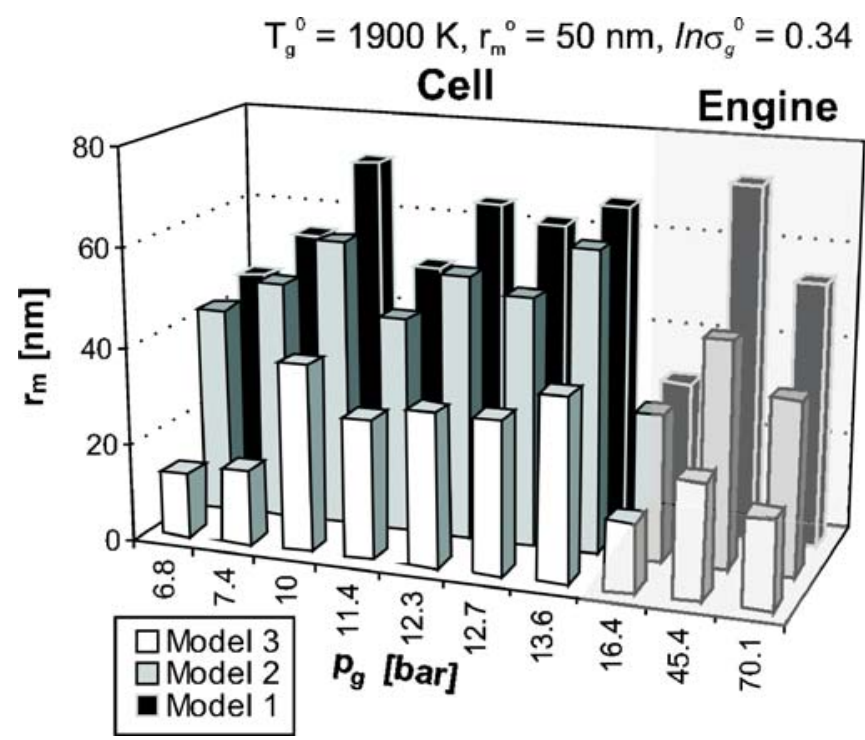

FIGURE 6 Best-fit mean particle radii $\left(r_{\mathrm{m}}\right)$ extracted from the least squares fitting of respective LII signal traces obtained at the indicated pressures in the constant volume cell (left 7 pressure readings) and in the Diesel engine (right 3 pressure readings). Results from using model functions 1-3 are distinguished by respective grayscale bars. The indicated initial-guess parameters $T_{\mathrm{g}}^{0}, r_{\mathrm{m}}^{0}$ and $\ln \sigma_{\mathrm{g}}^{0}$ were the same in all 3 cases

perature, PSD-function parameters, etc.). Since fitting such simple functions in a restricted time interval is computationally much more efficient than a full LSQ curve analysis, this approach seems appealing for particle sizing, especially when processing large amounts of data. For measurements in wellcharacterized environments Dankers et al. [25] showed that this strategy provides a one-to-one correspondence between the mean particle diameter and evaluated decay rate pairs. The extraction of particle size information from LII measurements at various pressures requires a large number of decay rate pairs $\left(k_{1}, k_{2}\right)$ to be determined in advance for a range of experimental conditions $\left(p_{\mathrm{g}}, T_{\mathrm{g}}, r_{\mathrm{m}}, \sigma\right)$. The significant variation of particle cooling rates for pressures covered in our experiments (see Fig. 4) also necessitates a suitable choice of time intervals from where to evaluate the LII signal decay. For our conditions it was necessary to precalculate more than $10^{4}$ decay rate pairs for storage in a library, which implies a considerable computational burden. As an example Fig. 7 reports contour-plots of corresponding early (upper panel) and late (lower panel) decay rates at a gas pressure of $0.7 \mathrm{MPa}$ and the particular case of a particle ensemble characterized with PSD-function parameters $r_{\mathrm{m}}$ and $\ln \sigma_{\mathrm{g}}=0.3$, respectively. The decay rates were obtained by fitting singleexponential decays to "best-fit" theoretical LII signal traces from environments characterized by the indicated range of gas temperature $T_{\mathrm{g}}$ and mean particle radius $r_{\mathrm{m}}$. It is seen that for fixed $T_{\mathrm{g}}$ (i.e., following a vertical line in each panel) the early decay rate $k_{1}$ (upper panel; evaluated in the range 5-100 ns) rapidly decreases with increasing particle radius, and exhibits little variation with particle size for the larger particles. On the contrary, depending on the gas temperature, the late decay rate $k_{2}$ (lower panel; evaluated here between 180 and $250 \mathrm{~ns}$ ) reaches a more or less pronounced maximum, after which it decreases with increasing particle radius. Since the true LII signal decay is not strictly exponential and only 
a limited number of $\left(T_{\mathrm{g}}, r_{\mathrm{m}}\right)$-parameters were evaluated, the depicted "decay rate surface" exhibits a somewhat irregular shape. The evaluated $k_{2}$ are close to zero for the small particle radii and the lower $T_{\mathrm{g}}$ due to the fact that for this parameter range the temperature gradient between soot particle and environment is large and the calculated LII signal profile almost disappeared, giving a zero slope within the chosen time interval.

We applied the "quick-fit" method in combination with a table look-up strategy to find size parameter values from experimental LII signal transients acquired in the constant volume combustion chamber at PSI. However, for two main reasons this strategy turned out to be ineffective. As was the case for the full LSQ curve analysis, because several physical variables such as gas temperature and the width of the PSD-function are not known as inputs to the model function, a search for appropriate $\left(k_{1}, k_{2}\right)$-pairs in a constrained parameter range is hardly possible. The small variation of the early decay rate with particle radius (upper panel in Fig. 7), further limits an unambiguous size determination by the search algorithm. Both constraints cause the table interpolation procedure to deliver ill-defined particle size parameter values.

\section{5}

\section{Discussion}

Our results suggest that in the LSQ-analysis a correlation exists between the obtained "best-fit" size parameter values and their respective "first-guess" values (see Figs. 5 and 6). This bias might have its origin in the topology of the multi-dimensional surface of squared residuals $\chi^{2}$ in (5) explored in the fitting algorithm. To illustrate this, Fig. 8a shows

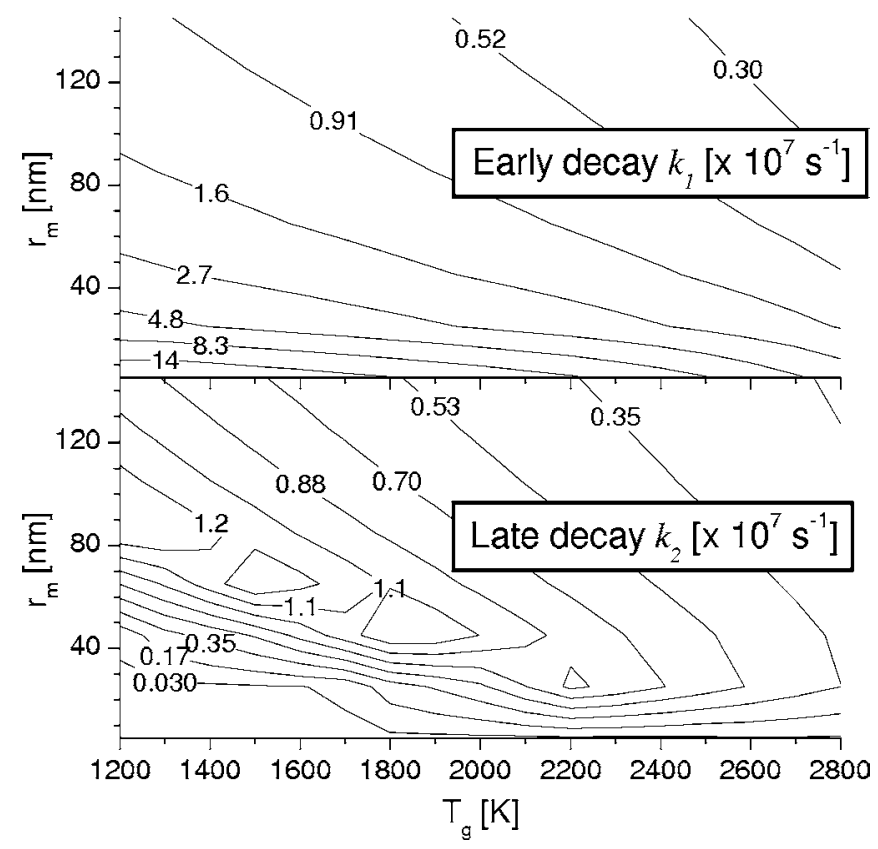

FIGURE 7 Kontour-plot of early (upper panel) and late (lower panel) decay rates determined by least-squares fitting of single-exponential decay functions to theoretical LII signal traces generated for experimentally relevant gas temperatures $\left(T_{\mathrm{g}}\right)$, a gas pressure of $0.7 \mathrm{MPa}$, and for a range of particle ensembles with mean particle radius $\left(r_{\mathrm{m}}\right)$ and a fixed size distribution width $\ln \sigma_{\mathrm{g}}=0.3$ the $\chi^{2}$-surface for a range of initial particle radii $r_{\mathrm{m}}^{0}$ and distribution widths $\sigma^{0}$ around some assumed "best-fit" values $\left(r_{\mathrm{m}}=10 \mathrm{~nm}, \sigma=0.3\right)$ when sub-model 3 is employed in the calculation of the respective LII time response profiles. Local minima exist on this surface next to the "best-fit" parameters (indicated on the bottom plane by broken lines). Depending on the optimization criterium $\left(\chi^{2} \leq \min\right.$.) chosen in the regression analysis, this topology will cause the optimization routine to end up in a local minimum closest to the parameters sampled in the neighborhood of the starting values, which causes the best-fit output values to be biased towards the chosen initial parameters. Interestingly, as shown in Fig. 8b, in the case that white noise is added to the calculated optimum LII signal response curve (5\% of the LII peak signal amplitude) these local minima become much less pronounced, and the minimization routine now samples a narrow flat valley on the $\chi^{2}$-surface.

It is obvious that the deduced primary particle sizes vary considerably from one model to the other (see Fig. 5), and are generally large if comparisons are made with the small amount of literature studies on engine in-cylinder [6-8] or

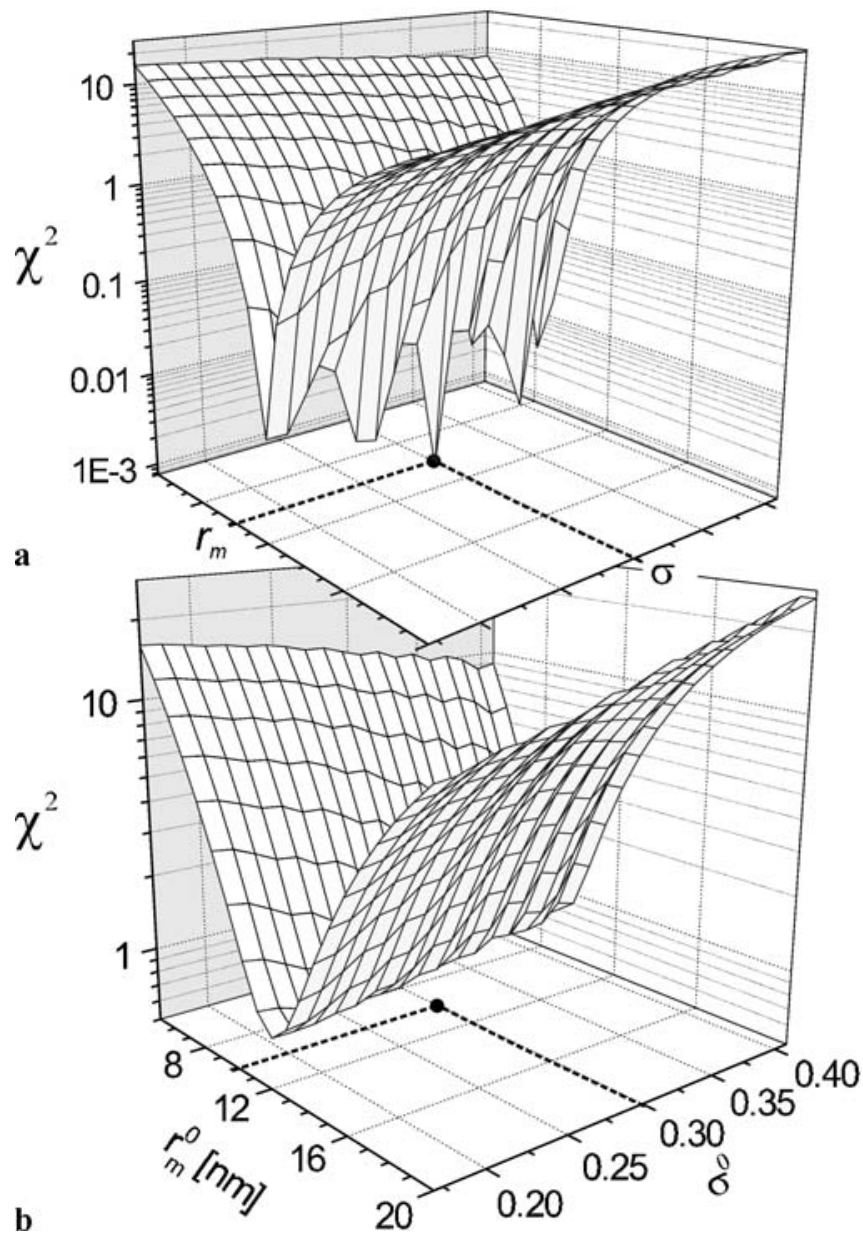

FIGURE 8 Global least-squares residual $\chi^{2}$ as function of the initial fitting variables mean particle radius $\left(r_{0}\right)$ and distribution width $(\sigma)$ calculated between a best-fit LII signal trace (with a mean particle radius of $r_{\mathrm{m}}=10 \mathrm{~nm}$ and logarithmic width of $\left.\sigma=\ln \sigma_{\mathrm{g}}=0.3\right)$ and those generated for a range of $\left(r_{\mathrm{m}}^{0}, \sigma^{0}\right)$-values close to the best-fit values: (a) smooth best-fit LII trace, (b) same as (a), with 5\% random noise added to the best-fit LII signal trace 
exhaust flow soot measurements [28], which report particle diameters between 30 and $80 \mathrm{~nm}$. However, as mentioned above, due to the inadequate adaptation of the employed submodels to high pressure combustion environments, combined with the poorly characterized sample environments we do not expect to provide quantitative results.

Another point of discussion is the use of suitable parameters in the submodel functions for generating the theoretical LII signal trace. In their original form the LII sub-models are only partially adapted for modeling the LII process in highpressure environments. As a consequence the energy loss terms due to heat conduction and vaporization in (2) should be evaluated in the continuum regime. However, sub-model 1 was originally set up for simulation of LII time profiles acquired in atmospheric pressure flames where a free molecular flow regime is acceptable. In sub-model 2 conductive cooling is calculated assuming a transition regime with $\alpha_{\mathrm{T}}=0.9$ and a temperature-dependent thermal conductivity. Only submodel 3 treats the pressure dependence in both heat flux terms (conduction and vaporization) by introducing correction factors which are functions of properly defined Knudsen numbers [29]. The limiting cases of high and low pressure are thus smoothly connected by a transition regime in the range of 0.1 to $2 \mathrm{MPa}$, also covered in our experiments. As a consequence, sub-model 3 delivers on average smaller particle diameters than obtained from the other two sub-models (see Fig. 6).

For reasons of consistency within the different models, in the present study soot is represented as a polydisperse size distribution of single spherical primary particles, a simplifying assumption which will certainly influence the calculated LII signal behavior. In real combustion environments experimental evidence shows that soot forms into aggregates composed of a polydisperse number distribution of spherical primary particles with a quite narrow size range [30,31]. Liu and coworkers investigated the shielding effect during cooling of laser-heated soot aggregates in ethylene diffusion flames [19] and found almost by a factor of two, a smaller cooling area for aggregates relative to soot consisting of single spherical particles [14].

\section{6}

\section{Conclusions}

In the present study ensemble-averaged soot particle sizing was performed by processing time-resolved laserinduced incandescence (TIRE-LII) signal transients obtained in turbulent, heavily sooting Diesel combustion processes at high pressure. Two evaluation strategies were implemented for the determination of mean particle diameters and size distribution widths: one is the commonly applied least squares fit of a theoretical LII signal curve to the measured one using a suitable model function as a solution of the energy and mass balance equations governing the particle heat-up and cooling phases. In the second approach the experimental LII signal intensity decay is piecewise approximated by singleexponential decay functions, and the fitted rates provide entry labels in a size parameter look-up table.

The current results show that good fits of the high-pressure LII profiles can be obtained using various model descriptions for the temperature decay, and appropriate estimates for the parameters characterizing the environment. The LII decay profiles do not contain sufficient structure to allow discrimination of individual models or parameter estimates. Therefore, it is of utmost importance to have independent knowledge of the relevant physical properties of both the soot and the environment, if the data are to be interpreted quantitatively. However, the trends observed as a function of global experimental conditions (notably pressure, in our case), are similar for all cases compared here. In particular, knowledge of both the temperature of the laser-heated particle as well as that of the ambient gas phase in the measurement volume is desirable. For measurements performed in unsteady or turbulent combusting flows the instantaneous determination of local gas temperature simultaneously with the LII transient remains a challenge. A better characterized sample environment would, however, narrow the parameter space being sampled in the course of the LSQ curve fit. Otherwise, some best-fit minimization condition (i.e., $\chi^{2} \leq \min$.) can then be easily satisfied during the multi-parameter regression analysis by tuning one or several unknown variables. As a consequence, depending on the initial start parameters biased best-fit parameter values may result.

For the quick-fit analysis tested with our experimental data the variation of evaluated decay rates with experimental parameters is too small for this technique to provide unambiguous particle size information, especially if noisy signal traces need to be analysed. In addition, if the probed environment can not be characterized well enough an extensive decay rate data base needs to be generated in advance, which compromises the methods speed advantage. This procedure may have to be repeated for situations where signal-to-noise considerations in the experimental data necessitate a choice of the individual time slots for signal trace evaluation different from those used in calculating the decay rate library.

In conclusion, in future applications of the TIRE-LII method for particle sizing in poorly characterized environments, data evaluation by more robust optimization procedures might be necessary for unambiguous target parameter identification. There also still remains a need for an improved, but tractable model description for application in high pressure LII diagnostics.

ACKNOWLEDGEMENTS The authors are thankful to L. Ganippa for active contributions during engine measurements, and to Prof. J.J. ter Meulen (RU) for constant interest in this collaborative work. We also acknowledge enlightening comments of one of the reviewers on the current status of soot morphology and its optical properties. The financial support of the Swiss Federal Office of Energy (BFE), the Swiss National Fund (SNF, contract \# 200021-107388/1) and the Technology Foundation STW in the Netherlands is gratefully acknowledged.

\section{REFERENCES}

1 L.A. Melton, Appl. Opt. 23, 2201 (1984)

2 R.J. Santoro, C.R. Shaddix, Laser-induced Incandescence. In: Applied Combustion Diagnostics, ed. by K. Kohse-Höinghaus, J.B. Jeffries (Taylor \& Francis, New York, 2002)

3 S. Schraml, S. Dankers, K. Bader, S. Will, A. Leipertz, Combust. Flame 120, $439(2000)$

4 P. Roth, A.V. Filippov, J. Aerosol Sci. 27, 95 (1996)

5 M. Hofmann, W.G. Bessler, C. Schulz, H. Jander, Appl. Opt. 12, 2052 (2003) 
6 B. Bougie, L. Ganippa, A.P. van Vliet, N.J. Dam, W.L. Meerts, J.J. ter Meulen, submitted to Combust. Flame (2005)

7 B.F. Kock, T. Eckhardt, P. Roth, Proc. Combust. Inst. 29, 2775 (2002)

8 J.E. Dec, P.L. Kelly-Zion, SAE Technical Paper Series 2000-01-0238, 23 (2000)

9 K. Inagaki, S. Takasu, K. Nakakita, SAE Technical Paper Series 199901-0508 (1999)

10 H. Bockhorn, H. Geitlinger, B. Jungfleisch, T. Lehre, A. Schön, T. Streibel, R. Suntz, Phys. Chem. Chem. Phys. 4, 3780 (2002)

11 T. Schittkowski, B. Mewes, D. Brüggemann, Phys. Chem. Chem. Phys. 4, 2063 (2002)

12 H.A. Michelsen, J. Chem. Phys. 118, 7012 (2003)

13 G.J. Smallwood, D.R. Snelling, F. Liu, Ö.L. Gülder, J. Heat Transf. 123, 814 (2001)

14 D.R. Snelling, F. Liu, G.J. Smallwood, Ö.L. Gülder, Combust. Flame 136, 180 (2004)

15 B. Öktem, M.P. Tolocka, B. Zhao, H. Wang, M.V. Johnston, Combust. Flame 142, 364 (2005)

16 M.M. Maricq, Combust. Flame 137, 340 (2004)

17 T.C. Fang, C.M. Megaridis, W.A. Sowa, G.S. Samuelson, Combust. Flame 112, 312 (1998)

18 Ü.Ö. Köylü, J.C. Xing, D.E. Rosner, Langmuir 11, 4848 (1995)
19 F. Liu, G.J. Smallwood, D.R. Snelling, J. Quantum. Spectrosc. Radiat. Transf. 93, 301 (2005)

20 A.V. Filippov, M. Zurita, D.E. Rosner, J. Colloid Interf. Sci. 229, 261 (2000)

21 A.V. Filippov, D.E. Rosner, Int. J. Heat Mass Transf. 43, 127 (2000)

22 Ü.Ö. Köylü, Combust. Flame 109, 488 (1997)

23 H. Bladh, P.-E. Bengtsson, Appl. Phys. B 78, 241 (2004)

24 MATLAB routines, MathWorks USA

25 S. Dankers, A. Leipertz, Appl. Opt. 43, 3726 (2004)

26 B. Bougie, M. Tulej, T. Dreier, N.J. Dam, T. Gerber, J.J. ter Meulen, Appl. Phys. B 80, 1039 (2005)

27 B. Bougie, L.C. Ganippa, A.P. van Vliet, K. Verbiezen, N.J. Dam, W.L. Meerts, J.J. ter Meulen, Proc. European Combustion Meeting (2005), p. 166

28 D.R. Snelling, G.J. Smallwood, R.A. Sawchuk, W.S. Neill, D. Gareau, D.J. Clavel, W.L. Chippior, F. Liu, Ö.L. Gülder, W.D. Bachalo, SAE Technical Paper Series 2000-01-1994 (2000)

29 M. Williams, S. Loyalka, Aerosol Science: Theory and Practice (Pergamon, Oxford, 1991)

30 B. Hu, B. Yang, Ü.Ö. Köylü, Combust. Flame 134, 93 (2003)

31 S.S. Krishnan, K.-C. Lin, G.M. Faeth, Int. J. Heat Mass Transf. 122, 517 (2000) 